\title{
Chylothorax: Abdominal approach
}

\author{
Daniel G. Nicastri, MD, and Raja M. Flores, MD
}

\footnotetext{
From the Department of Thoracic Surgery, Icahn School of Medicine at Mount Sinai, Mount Sinai Health System, New York, NY.

Disclosures: Authors have nothing to disclose with regard to commercial support.

Received for publication Sept 18, 2017; accepted for publication Sept 22, 2017; available ahead of print Oct 26, 2017.

Address for reprints: Raja M. Flores, MD, Department of Thoracic Surgery, Mount Sinai Health System, Icahn School of Medicine at Mount Sinai, One Gustave L. Levy Place, Box 1023, New York, NY 10029 (E-mail: raja.flores@mountsinai.org).

J Thorac Cardiovasc Surg 2018;155:814

$0022-5223 / \$ 36.00$

Copyright $(2017$ by The American Association for Thoracic Surgery

https://doi.org/10.1016/j.jtcvs.2017.09.111
}

Chylothorax is a serious complication with a historic mortality rate approaching $50 \%$, if high output. ${ }^{1}$ Today, lower mortality can be achieved, although the risk is still significant. ${ }^{2-4}$ Between pulmonary resections and esophagectomies, the thoracic surgeon will encounter this difficult complication and must devise a strategy for treatment. Diaz-Gutierrez and colleagues ${ }^{5}$ have presented their series of 3 laparoscopic ligations for refractory chylothorax after failure of other methods. They were successful in 2 of 3 patients, significantly decreasing chest tube output. The course of their patients demonstrates the severity of this problem. Three patients failed other methods before the laparoscopy. Two patients had chest tubes in for as long as 49 to 60 days along with complications ranging from intestinal bleeding to respiratory failure to death. The success of their well-reasoned plan for approaching the problem of chylothorax is very useful. The workup begins with lymphoscintigraphy and possible embolization, followed by consideration of thoracoscopic duct ligation, and finally laparoscopic cisterna chyli ligation. The authors highlighted the variability in anatomy of the lymphatic system and the potential utility of lymphoscintigraphy. On the other hand, this technique was not a novel approach but was nonetheless presented and described well. They do confess this limitation and note that other laparoscopic series also have small numbers.

In general, chylothorax predictors such as total daily drainage greater than $10 \mathrm{~mL} / \mathrm{kg}$, or greater than 1 liter daily, predict the likely failure of noninterventional management after the initiation of medical management., ${ }^{2,6}$ This management can include somatostatin, changing feeding to medium-chain triglycerides or elemental, or even nil per os. The biggest decision is discerning which ones will close spontaneously. Although operative management may cause the most somatic pain, it would be an understatement to say that conservative management with prolonged days in the hospital fasting is not a major morbidity in itself. However, it is still a complication

\section{References} Surgery. 1956;40:121-8. 3530-8.

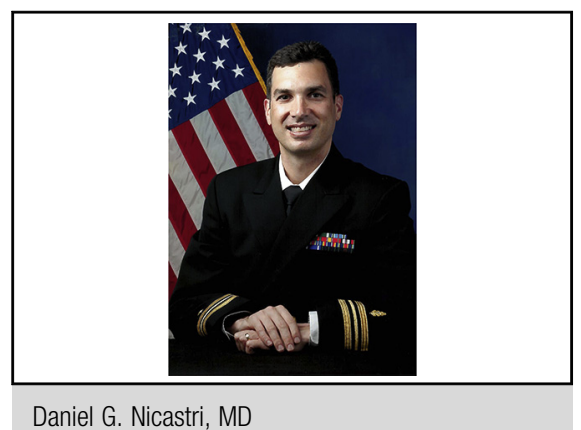

Central Message

Laparoscopic ligation of the cisterna chyli is a useful method for treating refractory chylothorax in select patients.

See Article page 815

associated with significant mortality risk. We advocate conservative management initially to best categorize the high versus low output leak. For those patients needing procedures, the algorithm presented by Diaz-Gutierrez and colleagues ${ }^{5}$ is not practical in many hospitals because interventionalists who perform lymphoscintigraphy are not always available. In those cases, we advocate thoracic duct ligation in select patients who can tolerate chest surgery or reoperative chest surgery. One advantage of ligation in the chest for unilateral chylothorax (using half-and-half milk from the operating room cafeteria before surgery through an orogastric or nasogastric tube) is that it can help identify the leak if sufficient time is allowed. This cannot be done easily with laparoscopic ligation of the cisterna chyli. However, when all else fails, this is a useful and elegant technique in select patients.

1. Maloney JV Jr, Spencer FC. The nonoperative treatment of traumatic chylothorax.

2. Shah RD, Luketich JD, Schuchert MJ, Christie NA, Pennathur A, Landreneau RJ, et al. Postesophagectomy chylothorax: incidence, risk factors, and outcomes. Ann Thorac Surg. 2012;93:897-904.

3. Marts BC, Naunheim KS, Fiore AC, Pennington DG. Conservative versus surgical management of chylothorax. Am J Surg. 1992;164:532-5.

4. Kranzfelder M, Gertler R, Hapfelmeier A, Friess H, Feith M. Chylothorax after esophagectomy for cancer: impact of the surgical approach and neoadjuvant treatment: systematic review and institutional analysis. Surg Endosc. 2013;27:

5. Diaz-Gutierrez I, Rao MV, Andrade RS. Laparoscopic ligation of cisterna chyli for refractory chylothorax: a case series and review to the literature. J Thorac Cardiovasc Surg. 2018;155:815-9.

6. Dugue L, Sauvanet A, Farges O, Goharin A, Le Mee J, Belghiti J. Output of chyle as an indicator of treatment for chylothorax complicating oesophagectomy. Br J Surg. 1998;85:1147-9. 